\section{OPEN ACCESS}

Edited by:

Wenjing Zhang,

Sichuan University, China

Reviewed by:

Jiajia Zhu,

The First Affiliated Hospital of Anhui

Medical University, China

Dongrong Xu,

Columbia University, United States

${ }^{*}$ Correspondence:

Hui Tang

tanghui2017@csu.edu.cn

Wenbin Guo

guowenbin76@csu.edu.cn

Specialty section:

This article was submitted to Neurocognitive Aging and Behavior,

a section of the journal

Frontiers in Aging Neuroscience

Received: 27 October 2021 Accepted: 30 December 2021

Published: 17 February 2022

Citation:

Pan P, Wang L, Wu C, Jin K, Cao S, Qiu Y, Teng Z, Li S, Shao T, Huang J, Wu H, Xiang H, Chen J, Liu F, Tang H and Guo W (2022)

Global Functional Connectivity Analysis Indicating Dysconnectivity of the Hate Circuit in Major

Depressive Disorder.

Front. Aging Neurosci. 13:803080

doi: 10.3389/fnagi.2021.803080

\title{
Global Functional Connectivity Analysis Indicating Dysconnectivity of the Hate Circuit in Major Depressive Disorder
}

\author{
Pan Pan', Lu Wang', Chujun Wu'1, Kun Jin', Song Cao', Yan Qiu'1, Ziwei Teng', \\ Sujuan Li ${ }^{1}$, Tiannan Shao ${ }^{1}$, Jing Huang ${ }^{1}$, Haishan Wu ${ }^{1}$, Hui Xiang ${ }^{1}$, Jindong Chen ${ }^{1}$, \\ Feng Liu ${ }^{2}$, Hui Tang ${ }^{1 *}$ and Wenbin Guo ${ }^{1,3 *}$
}

${ }^{1}$ National Clinical Research Center on Mental Disorders and Department of Psychiatry, The Second Xiangya Hospital of Central South University, Changsha, China, ${ }^{2}$ Department of Radiology, Tianjin Medical University General Hospital, Tianjin, China, ${ }^{3}$ Department of Psychiatry, The Third People's Hospital of Foshan, Foshan, China

Background: Abnormalities of functional connectivity (FC) in certain brain regions are closely related to the pathophysiology of major depressive disorder (MDD). Findings are inconsistent with different presuppositions in regions of interest. Our research focused on voxel-wise brain-wide FC changes in patients with MDD in an unbiased manner.

Method: We examined resting-state functional MRI in 23 patients with MDD and 26 healthy controls. Imaging data were analyzed by using global-brain FC (GFC) and used to explore the correlation of abnormal GFC values with clinical variables.

Results: Increased GFC values in the left medial superior frontal gyrus (SFGmed) and decreased GFC values in the right supplementary motor area (SMA) were observed in the patients with MDD compared with the controls. The decreased GFC values in the right SMA had a positive correlation with vitamin D and Hamilton Anxiety Scale (HAMA) scores.

Conclusion: Abnormal GFC in the hate circuit, particularly increased GFC in the left SFGmed and decreased GFC in the right SMA, appears to be a new sight for comprehending the pathological alterations in MDD.

Keywords: major depressive disorder, fMRI, global-brain functional connectivity, network, hate circuit

\section{INTRODUCTION}

Typical signs of major depressive disorder (MDD) include persistent negative emotions, reduced volitional activity, and cognitive dysfunction. MDD brings a heavy burden to the patients and their families due to its significant characteristics of high morbidity and mortality. However, the pathophysiology of MDD remains unclear. Several abnormal biochemical serum indices exhibit potential diagnostic significance for MDD. For example, the levels of some monoamine metabolites are significantly associated with the incidence of MDD (Richelson, 2001; Moret and Briley, 2011). 
Vitamin $\mathrm{D}$ is involved in the synthesis of monoamine neurotransmitters as a common fat-soluble vitamin. Previous studies found a significantly negative correlation between vitamin D levels in serum and MDD occurrence in participants (Hoang et al., 2011; Jääskeläinen et al., 2015). In other words, vitamin D deficiency might be involved in the incidence of MDD. Patients with MDD have a higher rate of vitamin D deficiency than the general population (Anglin et al., 2013; Gelaye et al., 2015). Lower levels of vitamin $\mathrm{D}$ in serum reduced neurotransmitters such as cholinergic, dopamine, and norepinephrine, thereby increasing the risk of MDD. Lower levels of vitamin D in older adults may be considered a biomarker for the depressive state (Adibfar et al., 2016).

Several previous studies on the pathogenesis for MDD have focused on genetic factors, social environment, and other aspects. Nonetheless, no single hypothesis could perfectly explain all the characteristics and prognosis of this disease. The development of brain imaging has provided a new sight and method for exploring the pathogenesis of MDD in recent years. Researchers have achieved important progress in probing the neurobiological mechanisms of MDD from the aspects of brain structure and function. Neuroimaging studies indicated that MDD may be caused by disruptions within discrete brain networks rather than abnormalities in isolated brain regions (Wang et al., 2012). Neural networks that have been studied extensively in patients with MDD include the default mode network (DMN) and frontolimbic network, where functional defects have been observed (Lee et al., 2008; Guo et al., 2015b; Scalabrini et al., 2020). Notably, the location of abnormally activated brain regions in previous studies focusing on brain dysfunction in MDD was involved in the main component of the hate circuit (Tao et al., 2013; Guo et al., 2015a). Hate, an intense basic human emotional response, plays an important role in psychological behavior and human evolution (Halperin, 2012). The hate circuit shows an altered activation when people watch stimuli they hate (Zeki and Romaya, 2008). Disgust at its core feeling of hate has been implicated in a wide range of psychological and mental conditions (Turnell et al., 2019). A number of studies have shown that hate is closely associated with depressive symptoms. For example, high levels of self-hate are directly related to the depressive state. Self-lathing in patients with MDD is associated with increased activity of the hate circuit (Surguladze et al., 2010; Turnell et al., 2019).

Resting-state functional connectivity (FC) between brain regions reflects its correlation with brain activity, which is an important tool to understand changes in FC between brain regions in patients with mood disorders. A number of studies have reported abnormal FC with inconsistent results in patients with MDD. For instance, patients with MDD in one study showed decreased FC between the medial orbitofrontal gyrus (Yu et al., 2021) and cerebellum (Zhu et al., 2020), whereas patients in another work exhibited increased homotopic FC (Liu et al., 2020) in the same brain regions.

One important factor contributing to these inconsistent results may be sample differences in age, population, locations, living style, etc. For example, patients with treatment-sensitive depression exhibited dysfunction in the frontoparietal top-down control network at specific frequency bands. However, patients with treatment-resistant depression showed abnormal FCs in the affective network, auditory network, visual network, and language processing cortex at the same frequency bands (He et al., 2016). Therefore, it is important to select first-episode, drugnaive patients with MDD to provide naive FC information to the pathophysiology of MDD.

Another important factor may be that previous studies focused on FC in specific brain regions. The region of interest (ROI) method was applied to these studies without a whole-brain examination. Critical areas associated with the core pathological alteration in MDD may be omitted by using this approach. Different ROI selections may result in different consequences. In this study, we adopted a data-driven approach, global-brain functional connectivity (GFC), to explore the pathophysiology of MDD and remedy the disadvantages of the ROI-based analysis (Preller et al., 2020). This method could be independent of the ROI selection. Therefore, GFC is helpful to obtain whole-brain FCs (Anticevic et al., 2014; Abdallah et al., 2017; Preller et al., 2018).

This study aims to utilize the GFC method, which belongs to the analysis method of the functional connectome, to probe the FC alterations in the whole brain of patients with MDD based on the aforementioned studies in MDD. We hypothesized that patients with MDD would exhibit GFC abnormalities in certain brain networks. Abnormal GFC in these brain regions may be related to the vitamin D levels in patients with MDD.

\section{MATERIALS AND METHODS}

\section{Participants}

A total of 28 first-episode, drug-naive patients with MDD were involved from the Inpatient Department of the Second Xiangya Hospital of Central South University. The age of patients was between 16 and 45 years. Each patient was diagnosed and screened by two psychiatric experts according to the Diagnostic and Statistical Manual of Mental Disorders, Fifth Edition (DSM-5) (First, 2013). The participants were righthanded. The enrolled patients were first-episode patients and with the course of the disease not exceeding 1 year. All patients were required to complete the Hamilton Depression Rating Scale17 (HAMD-17) (Hamilton, 1967), the Hamilton Anxiety Scale (Hamilton, 1959), and the Beck Depression Inventory-II (BDIII) (Steer, 1993) to evaluate the clinical sign of MDD. Meanwhile, the cognitive functions of all patients were evaluated by the Repeatable Battery for the Assessment of Neuropsychological Status (RBANS) (Randolph et al., 1998). The exclusion criteria for the participants included serious physical disease and any other mental disorders consistent with DSM-5, any history of alcohol or drug abuse, any drug treatment to MDD, lipidlowering therapy or vitamin D supplementation, pregnancy, and any contraindications to MRI scanning.

Thirty healthy controls who matched the patients with age, sex ratio, and education were involved by advertising in the local community. The DSM-5, Non-patients Version, was adopted to screen the healthy controls. The exclusion conditions 
included the history of serious neurological disorders, psychiatric disorders, and drugs or alcohol abuse in either the controls or their first-degree relatives.

All participants signed an informed consent form after obtaining an adequate explanation. Consent of the guardian was required for participants who aged under 18 years. This study is approved by the Ethics Committee of the Second Xiangya Hospital of Central South University and carried out in accordance with the Helsinki Declaration.

\section{Sample Collection}

To avoid circadian rhythms interfering with the data, fasting blood samples for biochemical analyses were collected between 7 a.m. and 9 a.m. for all participants. Liver and kidney function, blood glucose, lipid series, and vitamin D levels were analyzed by serum tests.

\section{Neuroimaging Data Acquisition and Preprocessing}

We used a Siemens 3.0 T scanner to obtain the resting-state images. The participants were required to lay on their back in a relaxed state and stay still during scanning. The neuroimaging data were automatically preprocessed using DPARBI and SPM8 in MATLAB (Yan et al., 2016). Detailed neuroimaging acquisition and preprocessing procedures are described in the Supplementary Material.

\section{Global-Brain FC Analysis}

Global-brain functional connectivity is a measurement tool that computes the mean time series correlation between a given voxel with every other voxel for an unbiased method to determine the location of abnormal FCs (Anticevic et al., 2013, 2014; Murrough et al., 2016; Pan et al., 2019, 2021). The gray matter mask is produced by using the gray matter probability map (probability $>0.2$ ) in the SPM8 software (Donishi et al., 2018). Such a threshold was chosen to eliminate the voxel with weak correlations that possibly originated from signal noises. A voxelbased GFC map was generated by composing GFC values of all voxels within the gray matter mask, where each voxel value represents the mean connectivity between the voxel and the rest of the brain. We computed the average Pearson's coefficient $(r)$ between the time series of each seed voxel and that of all other seed voxels throughout the whole brain after selecting each voxel within the gray matter mask as the seed voxel (Cole et al., 2010). GFC was calculated as average voxel-to-voxel connectivity throughout the gray matter mask as follows:

$$
\text { GFCa }=\sum_{\mathrm{b}=1}^{\mathrm{n}} \frac{\mathrm{r}\left(\mathrm{T}_{\mathrm{a}}, \mathrm{T}_{\mathrm{b}}\right)}{\mathrm{n}-1}
$$

where the Pearson correlation coefficient $(r)$ of time series Ts for a pair of given voxels a and b was calculated. Finally, the mean correlation coefficient within the gray matter mask of the entire brain was calculated using the MATLAB software (Scheinost et al., 2016; Pan et al., 2021), and the data were normalized and converted into $z$-values using Fisher $r$-to- $z$ transformation (Cole et al., 2010; Kaneoke et al., 2012; Thompson and Fransson, 2016).
The GFC maps between patients with MDD and controls were evaluated using two-sample $t$-tests. The framewise displacement (FD) values for each participant were calculated based on a previous study (Tozzi and Peters, 2017). The mean FD, education level, and age were substituted into the calculation as non-interest covariates. The threshold-free cluster enhancement (TFCE) was adopted to set the significance level as $p<0.05$, a strict multiple comparison correction strategy that could achieve the optimal balance between the family-wise error rate and test-retest reliability/repeatability (Chen et al., 2018).

\section{Functional Connectivity Within the Hate Circuit Analysis}

We performed a pairwise correlation analysis of six brain regions of the hate circuit in each subject by using the region-wise

TABLE 1 | Characteristics of the participants.

\begin{tabular}{|c|c|c|c|}
\hline & $\begin{array}{l}\text { Patients } \\
(n=23)\end{array}$ & $\begin{array}{l}\text { Controls } \\
(n=26)\end{array}$ & $P$-value \\
\hline Sex (male/female) & $7 / 16$ & $11 / 15$ & $0.390^{\mathrm{a}}$ \\
\hline Age (years) & $23.17 \pm 4.30$ & $20.85 \pm 3.13$ & $0.118^{\mathrm{b}}$ \\
\hline Education level (years) & $14.13 \pm 2.30$ & $14.62 \pm 2.06$ & $0.213^{b}$ \\
\hline HAMD-17 & $29.13 \pm 8.59$ & & \\
\hline HAMA-14 & $21.04 \pm 6.02$ & & \\
\hline BDI-II & $27.46 \pm 10.35$ & & \\
\hline VITD-T & $24.50 \pm 8.89$ & & \\
\hline Blood glucose & $3.69 \pm 0.86$ & & \\
\hline $\mathrm{TG}$ & $0.72 \pm 0.31$ & & \\
\hline $\mathrm{CHOL}$ & $3.75 \pm 0.92$ & & \\
\hline HDL-C & $1.61 \pm 1.79$ & & \\
\hline LDL-C & $2.08 \pm 0.83$ & & \\
\hline Vocabulary learning & $25.30 \pm 5.49$ & & \\
\hline Story retelling & $12.17 \pm 5.25$ & & \\
\hline Immediate memory total score & $37.48 \pm 9.51$ & & \\
\hline Graphic copy & $19.09 \pm 1.35$ & & \\
\hline Line positioning & $16.35 \pm 1.85$ & & \\
\hline Visual span total score & $35.43 \pm 2.04$ & & \\
\hline Picture named & $9.96 \pm 0.21$ & & \\
\hline Verbal fluency test & $18.35 \pm 4.98$ & & \\
\hline Verbal function total score & $28.30 \pm 4.97$ & & \\
\hline Digit span & $15.26 \pm 1.21$ & & \\
\hline Coding test & $51.04 \pm 11.42$ & & \\
\hline Attention total score & $66.30 \pm 11.55$ & & \\
\hline Vocabulary memory & $6.65 \pm 1.97$ & & \\
\hline Vocabulary recognition & $19.78 \pm 0.52$ & & \\
\hline Story recall & $6.70 \pm 2.89$ & & \\
\hline Figure memory & $15.61 \pm 2.64$ & & \\
\hline Delayed memory score & $48.74 \pm 6.36$ & & \\
\hline Stroop word & $98.30 \pm 10.36$ & & \\
\hline Stroop Color & $65.17 \pm 13.33$ & & \\
\hline Stroop Color-word & $39.04 \pm 7.38$ & & \\
\hline
\end{tabular}

a $\mathrm{p}$-value was obtained using a chi-square test.

bThe $p$-values were obtained using two-sample t-tests.

HAMD-17, Hamilton Depression Scale-17; HAMA, Hamilton Anxiety Scale; BDI-II, Baker Depression Inventory-II; VITD-T, vitamin D. 


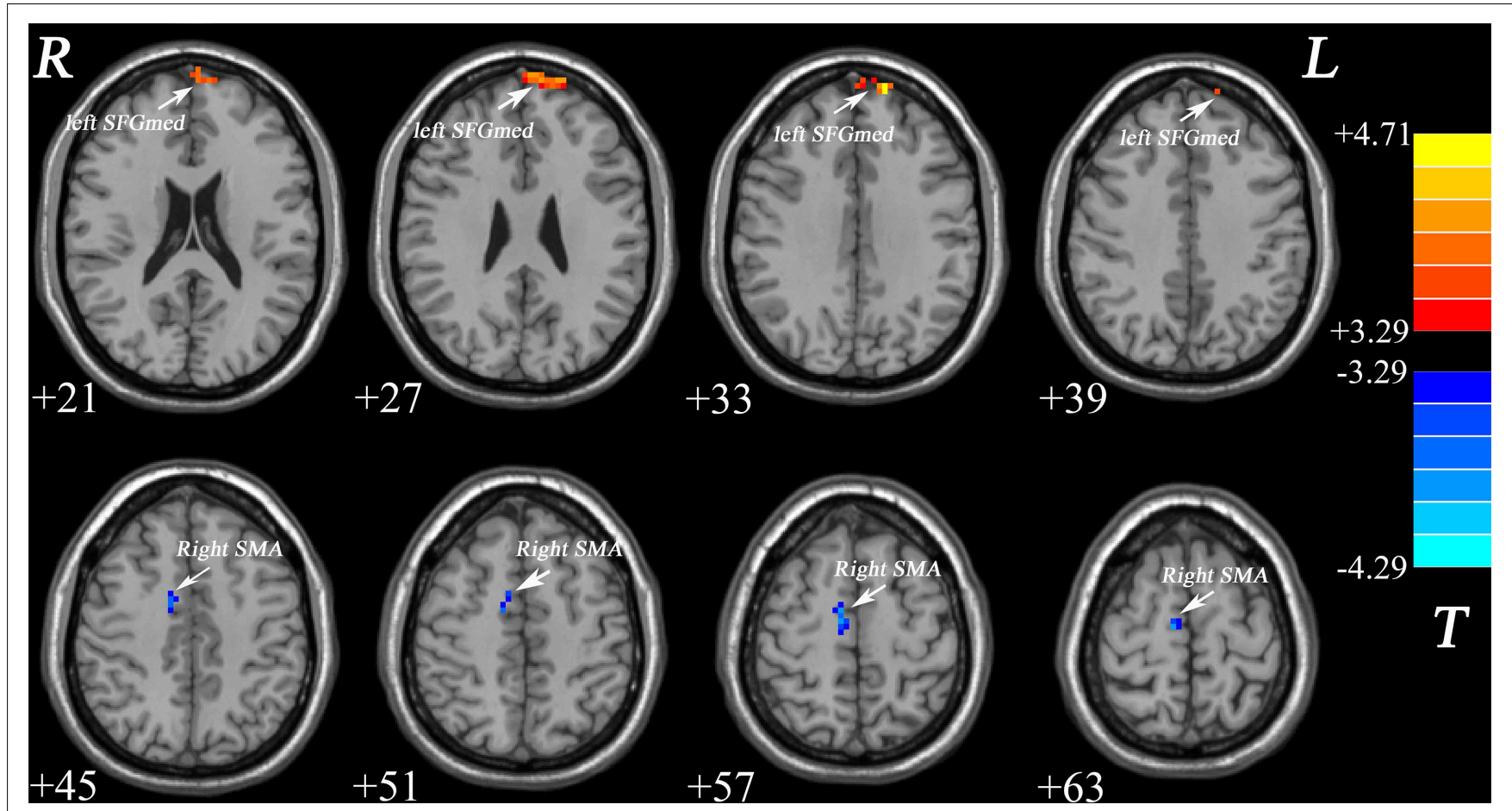

FIGURE 1 | Increased GFC values in the left SFGmed and decreased GFC values in the right SMA were observed in patients with MDD relative to healthy controls. GFC, global-brain functional connectivity; SFGmed, medial superior frontal gyrus; SMA, supplementary motor area; MDD, major depressive disorder.

FC method in the RESTplus software package and obtained correlation coefficient $r$. Fisher $r$-to- $z$ coefficients were adopted to transform into $z$-values to obtain a $6 \times 6 \mathrm{FC}$ matrix. The FC matrix between the patients with MDD and the controls was evaluated by two-sample $t$-tests.

\section{Statistical Analysis}

Two-sample $t$-tests were conducted on continuous variables. A chi-square test was performed for gender distribution. Pearson correlation analyses were performed between GFC values in patients with MDD and clinical characteristics including vitamin D, blood lipid series, and HAMD-17, HAMA, BDI-II, or RBANS scores. The significance level was Bonferroni corrected at $p<0.05$.

TABLE 2 | Significant differences in GFC values between groups.

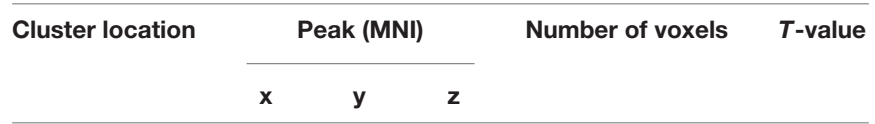

Patients > Controls

$\begin{array}{llllll}\text { Left SFGmed } & -15 & 63 & 33 & 64 & 4.6536\end{array}$

Patients $<$ Controls

Right SMA

$-15 \quad 63 \quad 33$

4.6536

MDD, major depressive disorder: GFC, global functional connectivity: SFGmed: medial superior frontal gyrus; SMA: supplementary motor area; MNI, Montreal Neurological Institute.

\section{RESULTS}

\section{Demographic and Clinical Characteristics}

Five patients and four controls were excluded because they had excessive head movement during functional MRI (fMRI) scans after preprocessing the neuroimaging data. The final sample included 23 patients with MDD and 26 healthy controls. No significant differences in sex ratio, age, and years of education were observed between patients with MDD and healthy controls. The demographic and clinical characteristics of the participants are summarized in Table $\mathbf{1 .}$

\section{Group Differences in Global-Brain FC}

As shown in Figure 1 and Table 2, patients with MDD exhibited decreased GFC values in the right supplementary motor area (SMA) $(t=-4.2841, p<0.001)$ and increased GFC values in the left medial superior frontal gyrus (SFGmed) $(t=4.6535$, $p<0.001)$ compared with the control group. No other differences were observed in the patients.

\section{Correlations Between Global-Brain FC and Clinical Characteristics}

Decreased GFC values in the right SMA were positively correlated with HAMA scores $(r=0.500, p=0.015)$ and vitamin $\mathrm{D}$ $(r=0.473, p=0.033$ ) (Figure 2). No significant correlations were found between the GFC values in the left SFGmed and the 


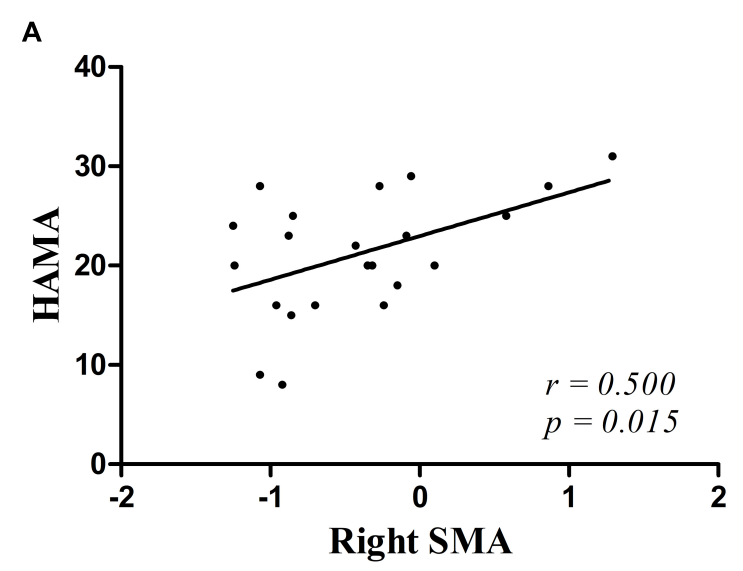

B

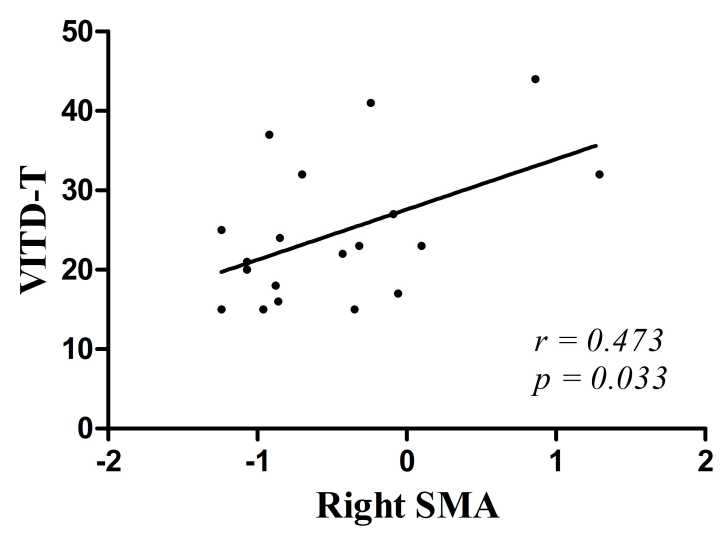

FIGURE 2 | (A,B) Positive correlations were observed between the GFC values in the right SMA and the scores of HAMA and the vitamin D in patients with MDD. HAMA, Hamilton Anxiety Scale; VITD-T, vitamin D.

clinical variables. No relationships between GFC and age, years of education, blood lipid series, and HAMD-17, BDI-II, or RBANS scores were observed.

\section{Functional Connectivity Difference Within the Hate Circuit}

Compared with controls, patients with MDD showed increased FC between the left insula and the left putamen, between the left putamen and the right putamen, and between the left SFG and the right SFG. FC between the right insula and the left SFG decreased (Figure 3).

\section{DISCUSSION}

Patients with MDD exhibit abnormal GFC in the brain regions of hate circuit in this study. Compared with the controls, significantly decreased GFC in the right SMA and increased GFC in the left SFGmed were observed in patients with MDD. The GFC in right SMA was positively correlated with vitamin D and the scores of HAMA.
Global-brain FC applies the basic image theory to study the overall connection between each voxel and every other voxel in the whole brain (Cole et al., 2010). This holistic approach was adopted to identify altered FC in the brain of patients with MDD and has proved to be very informative. This approach emphasizes qualitative data. Brain regions with abnormal FC may play a crucial role in coordinating massive brain activity patterns. Decreased GFC values may indicate low involvement of specific brain regions in diseases. Conversely, increased GFC values may indicate higher pathologies in specific brain regions (Buckner et al., 2009).

Superior frontal gyrus located in the upper prefrontal cortex (Shi et al., 2015) is considered to be composed of several subregions with different cellular structures (Petrides and Pandya, 1999, 2002), including Brodmann areas 6, 8, and 32 (Li et al., 2013). Previous studies on both task and resting-state fMRI have shown that different parts of SFG were involved in two anticorrelation networks, namely, task-positive network and DMN. These findings indicate that subregions exist in the SFG of humans (Li et al., 2013). Each subregion participates in different functional networks with its specific connectivity patterns of anatomical and functional levels. SFGmed plays as a pivotal node for DMN to participate in self-referential processing. The selfreferential processing refers to the process of encoding, storing, and extracting information closely related to self-experience, which is often accompanied by special affective meaning and motivation (Northoff et al., 2006). Increased GFC in the left SFGmed indicates that it is abnormally activated in this brain region of patients with $\mathrm{MDD}$ at rest compared with controls in this study. Abnormal activation in SFGmed was closely related to excessive self-focus under the influence of MDD. The excessive self-focus often takes the form of rumination, which is a common clinical symptom in patients with MDD. Previous neuroimaging studies have revealed that patients with MDD of abnormal FC in SFGmed were more likely to cause excessive self-focus when they encounter negative emotional events (Lemogne et al., 2012). The suppression was enhanced abnormally in irrelevant information outside the self in patients with MDD when they were excessively self-focused. This reactive mode aggravated negative emotion and eventually formed into negative automatic thoughts (Morawetz et al., 2016). Previous studies have exhibited that a deceptive reaction requires more complex cognitive processing or stronger mind control, which may involve cognitive controls, including cognitive inhibition, conflict monitoring, resolution, and generativity (Spence et al., 2001; Nuñez et al., 2005). SFGmed is the physiological basis for performing cognitive control (Sohn et al., 2007; Ursu et al., 2009; Pourtois et al., 2010; Xu et al., 2021). SFGmed plays a role in working memory processing, and abnormal FC in this region is the basis of the deterioration of working memory for patients with MDD (Vasic et al., 2008). Significant activation in the SFGmed was associated with feigned long-term memory impairment, suggesting that SFGmed may underlie the physiological basis for the execution of feigned memory (Chen et al., 2015). The most active brain region frequently observed during lying is SFG, indicating that the region is the basic neural circuit for lying (Kozel et al., 2004; Spence et al., 2004). Increased GFC in SFGmed suggests 

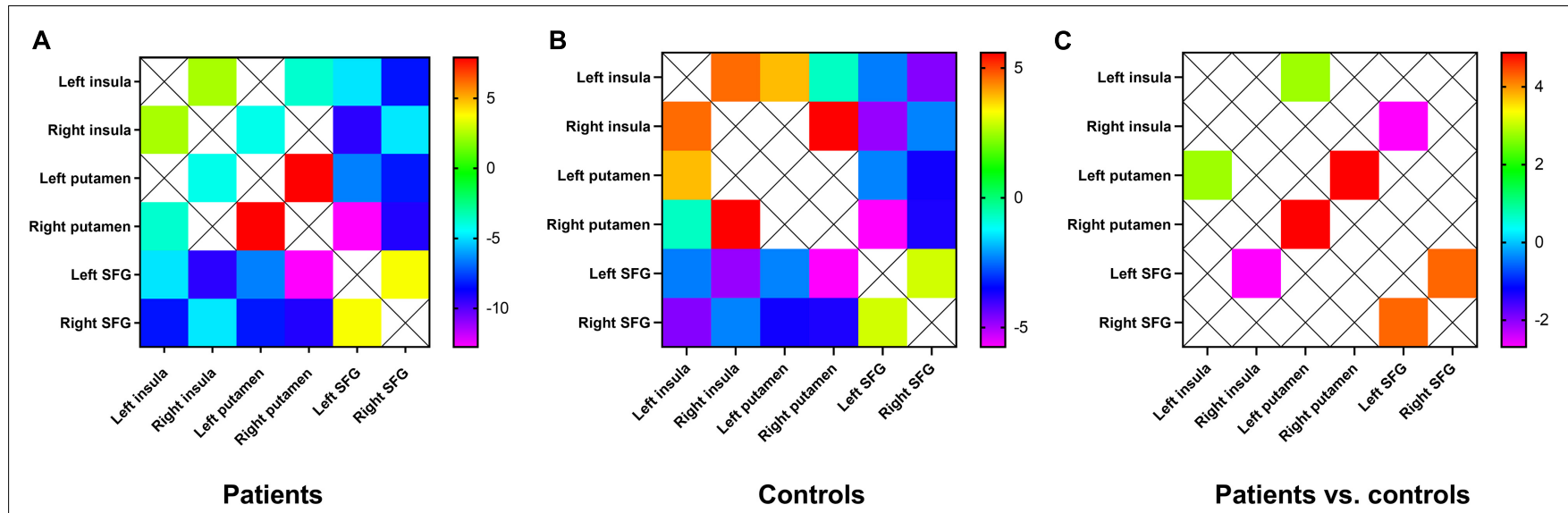

FIGURE 3 | (A) Mean FC between the brain regions of the hate circuit in the patient group. One-sample t-tests on the FCs were conducted. The warm color denotes higher FC values, and the cold color denotes lower FC in the patients. (B) Mean FC between the brain regions of the hate circuit in the control group. The range from red to green denotes higher FC values. Blue and purple denote lower FC. One-sample t-tests on the FCs were conducted. (C) Increased FC between the left insula and the right insula, between the left putamen and the right putamen, between the left SFG and the right SFG and decreased FC values in the right insula and the left SFG were observed in patients with MDD relative to healthy controls. FC, functional connectivity; SFG, superior frontal gyrus; MDD, major depressive disorder.

that compensatory activation occurs in this brain region affected by MDD in this study. Patients with MDD showed increased cognitive control during feigned memory disorders.

Supplementary motor area belongs to the posterior SFG, located in the medial side of hemispheres, which is mainly activated by motor tasks (Li et al., 2013). Generally, SMA takes part in controlled movement as the secondary motor cortex. A previous study has found significantly increased FC in SMA in patients with $\mathrm{MDD}$, suggesting that impaired function to this brain region may be involved in the pathophysiology of MDD (Frodl et al., 2010). This study reveals decreased GFC in the right SMA, which speculated spontaneous neural activity, and functional inhibition in severe depressive symptoms would cause thinking retardation, delayed speech, decreased initiative, and a series of involuntary motor behaviors such as akathisia and unconscious standing in several patients.

Tao et al. (2013) first reported FC alteration in the hate circuit of patients with MDD. They provided a perspective that the hate circuit may reflect reduced cognitive control over oneself and others in resentment generation. This study also focuses on FC within the hate circuit. The circuit includes the SFG, insula, and putamen. The SFG exhibited increased activities as well as enhanced activation in response to positive emotional stimuli. The putamen contains neurons in the preparation phase for motor behavior and participates in the perception of contempt and disgust. This brain region may have been involved in preparing offensive activity. The insula may be the physiological basis of activity in response to painful sensory stimuli. Damage to putamen and insula could affect the ability to recognize disgust signals in patients. The analysis of FC within the hate circuit exhibited abnormal FC between the SFG, putamen, and insula. Abnormal FC between these regions may be the physiological basis for the aggressive tendency and somatic discomfort in patients with MDD. Several studies have demonstrated that hate is closely associated with depressive symptoms (Overton et al., 2008; Surguladze et al., 2010). Reduced cognitive control in emotion is one of the important factors that cause MDD (Ebmeier et al., 2006). Patients with MDD have trouble in controlling negative thoughts. The hate circuit has been considered to be responsible for emotional and cognitive control regulation (Phillips et al., 2008; Fan et al., 2020). Impaired hate circuit might result in impaired cognitive control over pervasive internal self-loathing or hate of the external environment.

Decreased GFC values in the right SMA were positively correlated with vitamin D and HAMA scores. This finding indicates that decreased GFC in the right SMA may influence the anxiety level and provides theoretical foundation for the pathophysiology of anxiety signs in patients with MDD. Vitamin $\mathrm{D}$ deficiency has been shown to be causally associated with MDD (Milaneschi et al., 2014). Low vitamin D is closely related to the onset of MDD (Hoang et al., 2011), which probably explains that the treatment effect of MDD could be improved by adding vitamin $\mathrm{D}$ in addition to conventional antidepressant therapy. The cognitive function and spontaneous neural activity in patients with MDD may be affected by abnormal GFC in SMA due to alteration in vitamin $\mathrm{D}$.

Several constraints should be taken into account in this research. First, the difference in age may have a bias effect on the present results although age has been treated as a covariate. Second, the vitamin D level may vary between genders, but no significant difference was found in this research. Finally, the alterations in gray/white matter were not explored. Therefore, the mechanism of change in gray/white matter underlying GFC remains unclear.

\section{CONCLUSION}

Despite the limitation, this study presents that GFC abnormalities in the brain areas are associated with the hate circuit in patients with MDD. Abnormal GFC values in the right SMA might be one of the brain functional bases that cause alterations in the anxiety 
level. This study provides preliminary evidence that the vitamin $\mathrm{D}$ level in patients with MDD might be associated with abnormal FC in the hate circuit.

\section{DATA AVAILABILITY STATEMENT}

The original contributions presented in the study are included in the article/Supplementary Material, further inquiries can be directed to the corresponding authors.

\section{ETHICS STATEMENT}

The studies involving human participants were reviewed and approved by the Ethics Committee of the Second Xiangya Hospital of Central South University. The patients/participants provided their written informed consent to participate in this study.

\section{AUTHOR CONTRIBUTIONS}

WG, JC, HT, and HW provided the conception of the work. LW, CW, SL, YQ, ZT, KJ, and SC collected the data. FL, PP, TS, HX, and $\mathrm{JH}$ were responsible for the data analysis and interpretation. PP drafted the manuscript. WG critically revised the manuscript.

\section{REFERENCES}

Abdallah, C. G., Averill, L. A., Collins, K. A., Geha, P., Schwartz, J., Averill, C., et al. (2017). Ketamine Treatment and Global Brain Connectivity in Major Depression. Neuropsychopharmacology 42, 1210-1219. doi: 10.1038/npp.201 6.186

Adibfar, A., Saleem, M., Lanctot, K. L., and Herrmann, N. (2016). Potential Biomarkers for Depression Associated with Coronary Artery Disease: a Critical Review. Curr. Mol. Med. 16, 137-164. doi: 10.2174/1566524016666160126144143

Anglin, R. E., Samaan, Z., Walter, S. D., and McDonald, S. D. (2013). Vitamin D deficiency and depression in adults: systematic review and meta-analysis. $\mathrm{Br}$. J. Psychiatr. 202, 100-107. doi: 10.1192/bjp.bp.111.106666

Anticevic, A., Brumbaugh, M. S., Winkler, A. M., Lombardo, L. E., Barrett, J., Corlett, P. R., et al. (2013). Global prefrontal and fronto-amygdala dysconnectivity in bipolar I disorder with psychosis history. Biol. Psychiatr. 73, 565-573. doi: 10.1016/j.biopsych.2012.07.031

Anticevic, A., Hu, S., Zhang, S., Savic, A., Billingslea, E., Wasylink, S., et al. (2014). Global resting-state functional magnetic resonance imaging analysis identifies frontal cortex, striatal, and cerebellar dysconnectivity in obsessive-compulsive disorder. Biol. Psychiatr. 75, 595-605. doi: 10.1016/j.biopsych.2013.10.021

Buckner, R. L., Sepulcre, J., Talukdar, T., Krienen, F. M., Liu, H., Hedden, T., et al. (2009). Cortical hubs revealed by intrinsic functional connectivity: mapping, assessment of stability, and relation to Alzheimer's disease. J. Neurosci. 29, 1860-1873. doi: 10.1523/JNEUROSCI.5062-08.2009

Chen, X., Lu, B., and Yan, C. G. (2018). Reproducibility of R-fMRI metrics on the impact of different strategies for multiple comparison correction and sample sizes. Hum. Brain Mapp. 39, 300-318. doi: 10.1002/hbm.23843

Chen, Z. X., Xue, L., Liang, C. Y., Wang, L. L., Mei, W., Zhang, Q., et al. (2015). Specific marker of feigned memory impairment: the activation of left superior frontal gyrus. J. Forensic Leg. Med. 36, 164-171. doi: 10.1016/j.jflm.2015.09.008

Cole, M. W., Pathak, S., and Schneider, W. (2010). Identifying the brain's most globally connected regions. NeuroImage 49, 3132-3148. doi: 10.1016/j. neuroimage.2009.11.001
All authors contributed to the article and approved the final version of the manuscript.

\section{FUNDING}

This study was supported by grants from the National Natural Science Foundation of China (Grant Nos. 82171508, 81771447, and 81630033), the Natural Science Foundation of Hunan (Grant No. 2020JJ4784), the Science and Technology Program of Hunan Province (Grant No. 2020SK53413), the Commission Scientific Research Project of Hunan (Grant No. C2019164), and the Natural Science Foundation of Tianjin (Grant No. 18JCQNJC10900).

\section{ACKNOWLEDGMENTS}

We thank all individuals who served as the research participants.

\section{SUPPLEMENTARY MATERIAL}

The Supplementary Material for this article can be found online at: https://www.frontiersin.org/articles/10.3389/fnagi. 2021.803080/full\#supplementary-material

Donishi, T., Terada, M., and Kaneoke, Y. (2018). Effects of gender, digit ratio, and menstrual cycle on intrinsic brain functional connectivity: a whole-brain voxel-wise exploratory study using simultaneous local and global functional connectivity mapping. Brain Behav. 8:e00890. doi: 10.1002/brb3.890

Ebmeier, K., Rose, E., and Steele, D. (2006). Cognitive impairment and fMRI in major depression. Neurotox. Res. 10, 87-92. doi: 10.1007/BF03033237

Fan, Z., Yang, J., Zeng, C., Xi, C., Wu, G., Guo, S., et al. (2020). Bipolar Mood State Reflected in Functional Connectivity of the Hate Circuit: a Resting-State Functional Magnetic Resonance Imaging Study. Front. Psychiatr. 11:556126. doi: $10.3389 /$ fpsyt.2020.556126

First, M. (2013). Diagnostic and Statistical Manual of Mental Disorders, $5^{\text {th }}$ Edition, and Clinical Utility. J. Nerv. Ment. Dis. 201, 727-729. doi: 10.1097/nmd. 0b013e3182a2168a

Frodl, T., Bokde, A. L., Scheuerecker, J., Lisiecka, D., Schoepf, V., Hampel, H., et al. (2010). Functional connectivity bias of the orbitofrontal cortex in drugfree patients with major depression. Biol. Psychiatr. 67, 161-167. doi: 10.1016/j. biopsych.2009.08.022

Gelaye, B., Williams, M. A., Lemma, S., Berhane, Y., Fann, J. R., Vander Stoep, A., et al. (2015). Major depressive disorder and cardiometabolic disease risk among sub-Saharan African adults. Diabetes Metab. Syndr. 9, 183-191. doi: 10.1016/j.dsx.2014.05.003

Guo, W., Liu, F., Xiao, C., Zhang, Z., Liu, J., Yu, M., et al. (2015a). Decreased insular connectivity in drug-naïve major depressive disorder at rest. J. Affect. Dis. 179, 31-37. doi: 10.1016/j.jad.2015.03.028

Guo, W., Liu, F., Yu, M., Zhang, J., Zhang, Z., Liu, J., et al. (2015b). Decreased regional activity and network homogeneity of the fronto-limbic network at rest in naïvenaive major depressive disorder. Aus. N. Z. J. Psychiatr. 49, 550-556. doi: $10.1177 / 0004867415577978$

Halperin, E. (2012). The Nature of Hate. Peace confl. 18, 101-103.

Hamilton, M. (1959). The assessment of anxiety states by rating. Br. J. Med. Psychol. 32, 50-55. doi: 10.1111/j.2044-8341.1959.tb00467.x

Hamilton, M. (1967). Development of a rating scale for primary depressive illness. Br. J. Soc. Clin. Psychol. 6, 278-296. doi: 10.1111/j.2044-8260.1967.tb00 530.x 
He, Z., Cui, Q., Zheng, J., Duan, X., Pang, Y., Gao, Q., et al. (2016). Frequencyspecific alterations in functional connectivity in treatment-resistant and sensitive major depressive disorder. J. Psychiatr. Res. 82, 30-39. doi: 10.1016/ j.jpsychires.2016.07.011

Hoang, M. T., Defina, L. F., Willis, B. L., Leonard, D. S., Weiner, M. F., and Brown, E. S. (2011). Association between low serum 25-hydroxyvitamin D and depression in a large sample of healthy adults: the Cooper Center longitudinal study. Mayo Clin. Proc. 86, 1050-1055. doi: 10.4065/mcp.2011.0208

Jääskeläinen, T., Knekt, P., Suvisaari, J., Männistö, S., Partonen, T., Sääksjärvi, K., et al. (2015). Higher serum 25-hydroxyvitamin D concentrations are related to a reduced risk of depression. Br. J. Nutr. 113, 1418-1426. doi: 10.1017/ S0007114515000689

Kaneoke, Y., Donishi, T., Iwatani, J., Ukai, S., Shinosaki, K., and Terada, M. (2012). Variance and autocorrelation of the spontaneous slow brain activity. PLoS One 7:e38131. doi: 10.1371/journal.pone.0038131

Kozel, F. A., Revell, L. J., Lorberbaum, J. P., Shastri, A., Elhai, J. D., Horner, M. D., et al. (2004). A pilot study of functional magnetic resonance imaging brain correlates of deception in healthy young men. J. NeuroPsychiatr. Clin. Neurosci. 16, 295-305. doi: 10.1176/jnp.16.3.295

Lee, B. T., Seok, J. H., Lee, B. C., Cho, S. W., Yoon, B. J., Lee, K. U., et al. (2008). Neural correlates of affective processing in response to sad and angry facial stimuli in patients with major depressive disorder. Prog. Neuropsychopharmacol. Biol. Psychiatr. 32, 778-785. doi: 10.1016/j.pnpbp.2007. 12.009

Lemogne, C., Delaveau, P., Freton, M., Guionnet, S., and Fossati, P. (2012). Medial prefrontal cortex and the self in major depression. J. Affect. Dis. 136, e1-e11. doi: 10.1016/j.jad.2010.11.034

Li, W., Qin, W., Liu, H., Fan, L., Wang, J., Jiang, T., et al. (2013). Subregions of the human superior frontal gyrus and their connections. NeuroImage 78, 46-58. doi: 10.1016/j.neuroimage.2013.04.011

Liu, Y., Chen, Y., Liang, X., Li, D., Zheng, Y., Zhang, H., et al. (2020). Altered Resting-State Functional Connectivity of Multiple Networks and Disrupted Correlation With Executive Function in Major Depressive Disorder. Front. Neurol. 11:272. doi: 10.3389/fneur.2020.00272

Milaneschi, Y., Hoogendijk, W., Lips, P., Heijboer, A. C., Schoevers, R., van Hemert, A. M., et al. (2014). The association between low vitamin D and depressive disorders. Mol. Psychiatr. 19, 444-451. doi: 10.1038/mp.2013.36

Morawetz, C., Bode, S., Baudewig, J., Kirilina, E., and Heekeren, H. R. (2016). Changes in Effective Connectivity Between Dorsal and Ventral Prefrontal Regions Moderate Emotion Regulation. Cereb. Cortex 26, 1923-1937. doi: 10. 1093/cercor/bhv005

Moret, C., and Briley, M. (2011). The importance of norepinephrine in depression. NeuroPsychiatr. Dis. Treat. 7, 9-13. doi: 10.2147/NDT.S19619

Murrough, J. W., Abdallah, C. G., Anticevic, A., Collins, K. A., Geha, P., Averill, L. A., et al. (2016). Reduced global functional connectivity of the medial prefrontal cortex in major depressive disorder. Hum. Brain Mapp. 37, 32143223. doi: $10.1002 / \mathrm{hbm} .23235$

Northoff, G., Heinzel, A., de Greck, M., Bermpohl, F., Dobrowolny, H., and Panksepp, J. (2006). Self-referential processing in our brain-a meta-analysis of imaging studies on the self. NeuroImage 31, 440-457. doi: 10.1016/j. neuroimage.2005.12.002

Nuñez, J. M., Casey, B. J., Egner, T., Hare, T., and Hirsch, J. (2005). Intentional false responding shares neural substrates with response conflict and cognitive control. NeuroImage 25, 267-277. doi: 10.1016/j.neuroimage. 2004.10.041

Overton, P. G., Markland, F. E., Taggart, H. S., Bagshaw, G. L., and Simpson, J. (2008). Self-disgust mediates the relationship between dysfunctional cognitions and depressive symptomatology. Emotion 8, 379-385. doi: 10.1037/1528-3542. 8.3.379

Pan, P., Ou, Y., Su, Q., Liu, F., Chen, J., Zhao, J., et al. (2019). Voxel-based globalbrain functional connectivity alterations in first-epinaïvedrug-naive patients with somatization disorder. J. Affect. Dis. 254, 82-89. doi: 10.1016/j.jad.2019. 04.099

Pan, P., Qiu, Y., Teng, Z., Li, S., Huang, J., Xiang, H., et al. (2021). Increased Global-Brain Functional Connectivity Is Associated with Dyslipidemia and Cognitive Impairment in Firstnaïveode, Drug-Naive Patients with Bipolar Disorder. Neural Plast. 2021:5560453. doi: 10.1155/2021/5560453
Petrides, M., and Pandya, D. N. (1999). Dorsolateral prefrontal cortex: comparative cytoarchitectonic analysis in the human and the macaque brain and corticocortical connection patterns. Eur. J. Neurosci. 11, 1011-1036. doi: 10. 1046/j.1460-9568.1999.00518.x

Petrides, M., and Pandya, D. N. (2002). Comparative cytoarchitectonic analysis of the human and the macaque ventrolateral prefrontal cortex and corticocortical connection patterns in the monkey. Eur. J. Neurosci. 16, 291-310. doi: 10.1046/ j.1460-9568.2001.02090.x

Phillips, M. L., Ladouceur, C. D., and Drevets, W. C. (2008). A neural model of voluntary and automatic emotion regulation: implications for understanding the pathophysiology and neurodevelopment of bipolar disorder. Mol. Psychiatr. 13, 833-857. doi: 10.1038/mp.2008.65

Pourtois, G., Vocat, R., N'Diaye, K., Spinelli, L., Seeck, M., and Vuilleumier, P. (2010). Errors recruit both cognitive and emotional monitoring systems: simultaneous intracranial recordings in the dorsal anterior cingulate gyrus and amygdala combined with fMRI. Neuropsychologia 48, 1144-1159. doi: 10.1016/ j.neuropsychologia.2009.12.020

Preller, K. H., Burt, J. B., Ji, J. L., Schleifer, C. H., Adkinson, B. D., Stämpfli, P., et al. (2018). Changes in global and thalamic brain connectivity in LSD-induced altered states of consciousness are attributable to the 5-HT2A receptor. eLife 7:e35082. doi: 10.7554/eLife.35082

Preller, K. H., Duerler, P., Burt, J. B., Ji, J. L., Adkinson, B., Stämpfli, P., et al. (2020). Psilocybin Induces Time-Dependent Changes in Global Functional Connectivity. Biol. Psychiatr. 88, 197-207. doi: 10.1016/j.biopsych.2019.1 2.027

Randolph, C., Tierney, M. C., Mohr, E., and Chase, T. N. (1998). The Repeatable Battery for the Assessment of Neuropsychological Status (RBANS): preliminary clinical validity. J. Clin. Exp. Neuropsychol. 20, 310-319. doi: 10.1076/jcen.20.3. 310.823

Richelson, E. (2001). Pharmacology of antidepressants. Mayo Clin. Proc. 76, 511-527.

Scalabrini, A., Vai, B., Poletti, S., Damiani, S., Mucci, C., Colombo, C., et al. (2020). All roads lead to the default-mode network-global source of DMN abnormalities in major depressive disorder. Neuropsychopharmacology 45, 2058-2069. doi: 10.1038/s41386-020-0785-x

Scheinost, D., Tokoglu, F., Shen, X., Finn, E. S., Noble, S., Papademetris, X., et al. (2016). Fluctuations in Global Brain Activity Are Associated With Changes in Whole-Brain Connectivity of Functional Networks. IEEE Trans. Biomed. Eng. 63, 2540-2549. doi: 10.1109/TBME.2016.2600248

Shi, Y., Zeng, W., Wang, N., Wang, S., and Huang, Z. (2015). Early warning for human mental sub-health based on fMRI data analysis: an example from a seafarers' resting-data study. Front. Psychol. 6:1030. doi: 10.3389/fpsyg.2015. 01030

Sohn, M. H., Albert, M. V., Jung, K., Carter, C. S., and Anderson, J. R. (2007). Anticipation of conflict monitoring in the anterior cingulate cortex and the prefrontal cortex. Proc. Natl. Acad. Sci. U S A 104, 10330-10334. doi: 10.1073/ pnas.0703225104

Spence, S. A., Farrow, T. F., Herford, A. E., Wilkinson, I. D., Zheng, Y., and Woodruff, P. W. (2001). Behavioural and functional anatomical correlates of deception in humans. Neuroreport 12, 2849-2853. doi: 10.1097/00001756200109170-00019

Spence, S. A., Hunter, M. D., Farrow, T. F., Green, R. D., Leung, D. H., Hughes, C. J., et al. (2004). A cognitive neurobiological account of deception: evidence from functional neuroimaging. Philos. Trans. Phys. Sci. Eng. 359, 1755-1762. doi: 10.1098/rstb.2004.1555

Steer, R. (1993). Manual for the Beck Depression Inventory-II. Agra: Psychological Corporation.

Surguladze, S. A., El-Hage, W., Dalgleish, T., Radua, J., Gohier, B., and Phillips, M. L. (2010). Depression is associated with increased sensitivity to signals of disgust: a functional magnetic resonance imaging study. J. Psychiatr. Res. 44, 894-902. doi: 10.1016/j.jpsychires.2010.02.010

Tao, H., Guo, S., Ge, T., Kendrick, K. M., Xue, Z., Liu, Z., et al. (2013). Depression uncouples brain hate circuit. Mol. Psychiatr. 18, 101-111. doi: 10.1038/mp.201 1.127

Thompson, W. H., and Fransson, P. (2016). On Stabilizing the Variance of Dynamic Functional Brain Connectivity Time Series. Brain Connectivity 6, 735-746. doi: 10.1089/brain.2016.0454 
Tozzi, A., and Peters, J. F. (2017). From abstract topology to real thermodynamic brain activity. Cogn. Neurodyn. 11, 283-292. doi: 10.1007/s11571-017-9 431-7

Turnell, A. I., Fassnacht, D. B., Batterham, P. J., Calear, A. L., and Kyrios, M. (2019). The Self-Hate Scale: development and validation of a brief measure and its relationship to suicidal ideation. J. Affect. Dis. 245, 779-787. doi: 10.1016/j.jad. 2018.11.047

Ursu, S., Clark, K. A., Aizenstein, H. J., Stenger, V. A., and Carter, C. S. (2009). Conflict-related activity in the caudal anterior cingulate cortex in the absence of awareness. Biol. Psychol. 80, 279-286. doi: 10.1016/j.biopsycho.2008.10.008

Vasic, N., Walter, H., Sambataro, F., and Wolf, R. C. (2008). Aberrant functional connectivity of dorsolateral prefrontal and cingulate networks in patients with major depression during working memory processing. Psychol. Med. 39, $977-$ 987. doi: 10.1017/S0033291708004443

Wang, L., Hermens, D. F., Hickie, I. B., and Lagopoulos, J. (2012). A systematic review of resting-state functional-MRI studies in major depression. J. Affect. Dis. 142, 6-12. doi: 10.1016/j.jad.2012.04.013

Xu, Y., Zhang, X., Xiang, Z., Wang, Q., Huang, X., Liu, T., et al. (2021). Abnormal Functional Connectivity Between the Left Medial Superior Frontal Gyrus and Amygdala Underlying Abnormal Emotion and Premature Ejaculation: a Resting State fMRI Study. Front. Neurosci. 15:704920. doi: 10.3389/fnins.2021. 704920

Yan, C. G., Wang, X. D., Zuo, X. N., and Zang, Y. F. (2016). DPABI: data Processing \& Analysis for (Resting-State) Brain Imaging. Neuroinformatics 14, 339-351. doi: 10.1007/s12021-016-9299-4

Yu, R., Tan, H., Peng, G., Du, L., Wang, P., Zhang, Z., et al. (2021). Anomalous functional connectivity within the default-mode nnaïvek in treatment-naive patients possessing first-episode major depressive disorder. Medicine 100:e26281. doi: 10.1097/MD.000000000002 6281

Zeki, S., and Romaya, J. P. (2008). Neural correlates of hate. PLoS One 3:e3556. doi: 10.1371/journal.pone.0003556

Zhu, D. M., Yang, Y., Zhang, Y., Wang, C., Wang, Y., Zhang, C., et al. (2020). Cerebellar-cerebral dynamic functional connectivity alterations in major depressive disorder. J. Affect. Dis. 275, 319-328. doi: 10.1016/j.jad.2020. 06.062

Conflict of Interest: The authors declare that the research was conducted in the absence of any commercial or financial relationships that could be construed as a potential conflict of interest.

Publisher's Note: All claims expressed in this article are solely those of the authors and do not necessarily represent those of their affiliated organizations, or those of the publisher, the editors and the reviewers. Any product that may be evaluated in this article, or claim that may be made by its manufacturer, is not guaranteed or endorsed by the publisher.

Copyright (c) 2022 Pan, Wang, Wu, Jin, Cao, Qiu, Teng, Li, Shao, Huang, Wu, Xiang, Chen, Liu, Tang and Guo. This is an open-access article distributed under the terms of the Creative Commons Attribution License (CC BY). The use, distribution or reproduction in other forums is permitted, provided the original author(s) and the copyright owner(s) are credited and that the original publication in this journal is cited, in accordance with accepted academic practice. No use, distribution or reproduction is permitted which does not comply with these terms. 